\title{
A Case Study in Noncommutative Topology
}

\author{
Claude L. Schochet \\ Communicated by Gerald B. Folland
}

ABSTRACT. This is an expository note focused upon one example, the irrational rotation $C^{*}$-algebra. We discuss how this algebra arises in nature-in quantum mechanics, group actions, and foliated spaces, and we explain how $K$-theory is used to get information out of it.

\section{Introduction}

This is the opposite of a survey paper. Here we are interested in one example, usually known as the irrational rotation $C^{*}$-algebra or noncommutative torus and written $A_{\lambda}$, where $\lambda$ is some irrational number between 0 and 1 . We will show that $A_{\lambda}$ arises in at least three quite different contexts:

(1) quantum mechanics,

(2) action of a group on a compact Hausdorff space,

(3) foliated spaces.

Then we will use $K$-theory and traces to show that for $\lambda$ irrational between 0 and $1 / 2$, the $A_{\lambda}$ are all nonisomorphic.

A word about the title. Commutative $C^{*}$-algebras with unit ("unital") and compact Hausdorff spaces are equivalent categories: given a compact Hausdorff space $X$ you can form $C(X)$, the commutative unital $C^{*}$-algebra of all continuous complex-valued functions on $X$, and given a commutative unital $C^{*}$-algebra $A$, its maximal ideal

Claude (Chaim) Schochet is visiting professor of mathematics at the Technion, in Haifa, Israel, having retired emeritus from Wayne State University. His email address is c1 smath@gmai 1 . com.

It is a pleasure to thank Marc Rieffel who, besides contributing the most important theorems to this note, was very helpful in its preparation, and to thank my old friend and co-author Jonathan Rosenberg.

For permission to reprint this article, please contact: reprint-permission@ams.org.

DOI: http://dx.doi.org/10.1090/noti1684 space $X$ is compact Hausdorff and $A \cong C(X)$. So studying commutative unital $C^{*}$-algebras is the same as studying compact Hausdorff spaces-a natural category for algebraic topology. Most $C^{*}$-algebras are noncommutative, and so studying them is doing noncommutative topology!

Our goal is to write as if we are sitting in a coffeehouse and explaining an idea to a good friend (on napkins, of course). So we
Mathematicians

$$
\begin{aligned}
& \text { struggled to keep } \\
& \text { up, to keep the } \\
& \text { physics honest. }
\end{aligned}
$$
are interested in getting an idea across but not at all interested in the technical details that, in any event, would be lost if the coffee spilled. ${ }^{1}$

\section{Quantum Mechanics}

In 1926-1927 the quantum-mechanical revolution in physics changed our understanding of the world. As has been the pattern since, the physicists knew what they wanted, and the mathematicians struggled to keep up, to keep the physics honest (as a mathematician would put it).

The simplest model of the hydrogen atom revolved about two self-adjoint operators $P$ and $Q$ that were to measure position and momentum of the electron. Heisenberg and Born showed that if $Q$ is the position operator and $P$ the momentum operator, then we have the canonical commutation relation

$$
P Q-Q P=-i \hbar I,
$$

where $\hbar$ is Planck's constant.

\footnotetext{
${ }^{1}$ I learned this technique from Dror Bar-Natan, who gave a great colloquium talk entitled "From Stonehenge to Witten, Skipping all the Details."
} 
It is easy to see that there are no $n \times n$ matrices $P$ and $Q$ such that

$$
P Q-Q P=-i \hbar I
$$

with $\hbar \neq 0$ : just observe that

$$
\operatorname{tr}(P Q-Q P)=\operatorname{tr}(P Q)-\operatorname{tr}(Q P)=0,
$$

but $\operatorname{tr}(-\hbar I)=-i n \hbar$. It is not much harder to see that there are no bounded self-adjoint operators on a Hilbert space with this property. There are unbounded ones, but to avoid technicalities with such operators it is best to pass to the corresponding one-parameter unitary groups

$$
U_{s}=e^{i s P}, \quad V_{t}=e^{i t Q},
$$

for which the commutation relation $P Q-Q P=-i \hbar$ becomes

$$
U_{s} V_{t}=e^{-i \hbar s t} V_{t} U_{s} .
$$

This is called the Weyl form of the canonical commutation relation. Such pairs of unitary groups exist: for example, $U_{s} f(x)=f(x-\hbar s)$ and $V_{t} f(x)=e^{i t x} f(x)$ on $L^{2}(\mathbb{R})$. Moreover, by the Stone-von Neumann theorem they are all unitarily equivalent, subject to an irreducibility condition. Taking $s=t=1$ and $\lambda=-2 \pi \hbar$, we obtain unitary operators $U$ and $V$ such that

$$
U V=e^{2 \pi i \lambda} V U \text {. }
$$

These operators are bounded operators on the same Hilbert space, $U, V \in \mathcal{B}(\mathcal{H})$. So we may take the (noncommuting) polynomial algebra generated by $U, V$, and their adjoints. We then close up this algebra with respect to the operator norm and reach our goal, the $C^{*}$-algebra $A_{\lambda}$, constructed visibly as a norm-closed, $*$-closed subalgebra of $B(\mathcal{H})$.

This is the first construction of the $A_{\lambda}$. We may restrict attention to $\lambda \in[0,1)$ and ask an elementary question: as $\lambda$ changes, how is $A_{\lambda}$ affected? It turns out that the case of greatest interest is when $\lambda$ is irrational, and so we will restrict to that case as needed.

\section{Homeomorphisms of the Circle}

Let $\phi: S^{1} \rightarrow S^{1}$ be rotation of the circle by $2 \pi \lambda$ radians counterclockwise. Any rotation is a homeomorphism and thus determines an action of the integers on the circle by sending $n$ to $\phi^{n}$. This defines an action of the integers on $C\left(S^{1}\right)$ and from this one can construct a $C^{*}$-algebra

$$
C\left(S^{1}\right) \rtimes \mathbb{Z}
$$

as follows. ${ }^{2}$

For $\lambda=0$ or $1, C\left(S^{1}\right) \rtimes \mathbb{Z}$ is simply $C\left(T^{2}\right)$, continuous functions on the torus. For $\lambda$ irrational we can realize this algebra as follows. Take $\mathcal{H}$ to be the Hilbert space $L^{2}\left(S^{1}\right)$ and let $T \in \mathcal{B}(\mathcal{H})$ be the bounded invertible operator $T f=f \circ \phi$. Any $f \in C\left(S^{1}\right)$ gives a pointwise multiplication operator $M_{f} \in \mathcal{B}(\mathcal{H})$. Then $C\left(S^{1}\right) \rtimes \mathbb{Z}$ is

\footnotetext{
${ }^{2}$ This is actually a quite general construction. Starting with any $C^{*}$-algebra and locally compact group $G$ acting on $A$ you get a new crossed-product $C^{*}$ algebra built by taking the algebraic crossed product and then completing. There are some topological choices for how the completion is done, but if the group is commutative then the completions coincide.
}

the norm-closed $*$-algebra generated by $T$ and by all of the $M_{f}$. Note that finite sums of the form

$$
\sum_{n=-k}^{k} M_{f_{n}} T^{n}
$$

are dense in $C\left(S^{1}\right) \rtimes \mathbb{Z}$. For $\lambda$ irrational there is a unique normalized trace ${ }^{3} \tau$ on $C\left(S^{1}\right) \rtimes \mathbb{Z}$ given on finite sums by

$$
\tau\left(\sum_{n} M_{f_{n}} T^{n}\right)=\int_{S^{1}} f_{0}(t) d t \in \mathbb{R}
$$

where $d t$ is normalized Lebesgue measure on the circle. It is not at all hard to prove that if $\lambda$ is irrational, so that the action of $\mathbb{Z}$ on the circle is free, then

$$
A_{\lambda} \cong C\left(S^{1}\right) \rtimes \mathbb{Z} \text {. }
$$

\section{Foliated Spaces}

The local picture of a foliated space is $\mathbb{R}^{p} \times N$, where $N$ is some topological space. A subset of the form $\mathbb{R}^{p} \times\{n\}$ is called a plaque and a measurable subset $T \subseteq \mathbb{R}^{p} \times N$ which meets each plaque at most countably often (the simplest being $\{x\} \times N)$ is called a transversal. The global picture is more complicated. We say that a (typically compact Hausdorff) space $X$ is a foliated space if each point in $X$ has an open neighborhood homeomorphic to the local picture and locally the plaques fit together smoothly. A leaf is a maximal union of overlapping plaques; by construction it is a smooth $p$-dimensional manifold.

Here is an example. Start with the unit square $[0,1] \times$ $[0,1]$ foliated by the vertical lines $\{x\} \times[0,1]$. Glue the left and right sides of the square together-that is, identify $(0, t)$ with $(1, t)$-to make a cylinder, again foliated by vertical lines. If we now glue the top and bottom of the cylinder together, identifying $(x, 0)$ with $(x, 1)$ we get a torus foliated by circles that go around the central hole. (See Figure 1.)

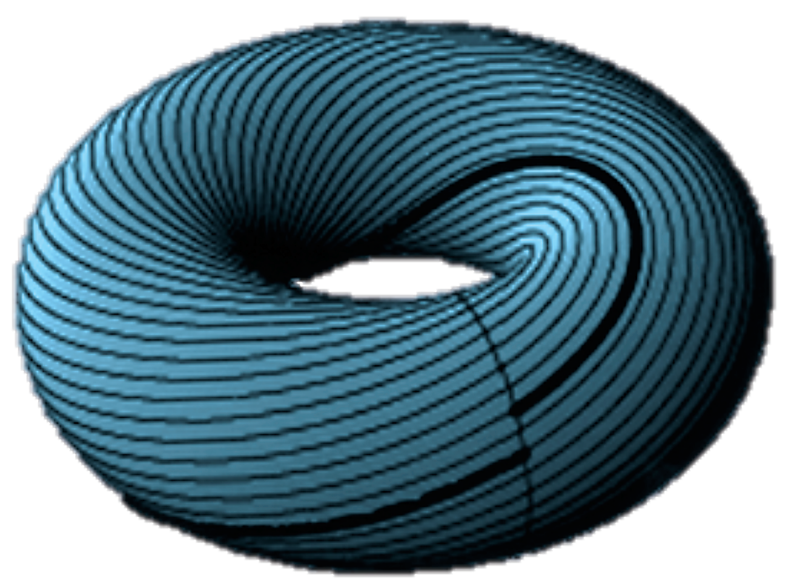

Figure 1. A torus foliated by lines of given irrational slope gives rise to a $C^{*}$-algebra.

To get something more interesting, we can give the cylinder a twist before gluing the ends together: we glue

${ }^{3} A$ normalized trace on $A$ is a linear functional on $A$ such that $\tau\left(x^{*} x\right)=\tau\left(x x^{*}\right) \geq 0$ for all $x$ and $\tau(1)=1$. 
$(x, 0)$ to $(x \oplus \lambda, 1)$, where “ $\oplus$ " means addition $\bmod 1$. Now something quite unusual happens, and here we must specify whether $\lambda$ is rational or not. If $\lambda$ is rational, then each leaf of the foliated space is actually a circle wrapped around the torus several times. On the other hand, if $\lambda$ is irrational, then you do not get circles: every leaf is a line $\mathbb{R}$. Furthermore, as in Figure 1, each line is wrapped about the torus infinitely often: each line is actually dense in the torus. This construction is called the Kronecker flow on the torus.

Every foliated space satisfying very minimal technical assumptions has a $C^{*}$-algebra associated to it. This

\section{There is a natural trace that arises.}

is due to $\mathrm{H}$. E. Winkelnkemper (1983) and to A. Connes (1979). In our context these always have the form $A \otimes \mathcal{K}$ where $\mathcal{K}$ is the $C^{*}$-algebra of the compact operators. ${ }^{4}$ Here are some examples:

(1) If $X=F \times B$ or more generally, if $X$ is the total space of a compact fibre bundle with fibre some smooth manifold $F$ and base the compact Hausdorff space $B$, then $X$ is foliated by the fibres and the foliation algebra is $C(B) \otimes \mathcal{K}$.

(2) If $X$ is the torus foliated by circles, then it is a fibre bundle of the form $S^{1} \rightarrow X \rightarrow S^{1}$ and by (1) the foliation algebra is $C\left(S^{1}\right) \otimes \mathcal{K}$ (where the $S^{1}$ in $C\left(S^{1}\right)$ is the base of the fibre bundle, not the generic fibre).

(3) (the punch line) If $X$ is the Kronecker flow on the torus, then the foliation algebra is $A_{\lambda} \otimes \mathcal{K}$.

Note for $0<\lambda<1 / 2$ that using $\lambda$ or $1-\lambda$ gives the same foliated space, and so $A_{\lambda} \cong A_{1-\lambda}$. Thus, we restrict attention to irrational $\lambda$ between 0 and $1 / 2$.

There is a natural trace that arises in this construction as well. What is needed is an invariant transverse measure. A transverse measure measures transversals, naturally enough. If it has enough nice properties then it is an invariant transverse measure. Not all foliated spaces have them, but the ones we are looking at do. In the case of the fibre bundle above, the foliation algebra is simply $C(B) \otimes \mathcal{K}$ and invariant transverse measures correspond to certain measures on $B$. Invariant transverse measures correspond to Ruelle-Sullivan currents in foliation theory (cf. [1] Ch. IV).

In the case of the Kronecker flow on the torus, the invariant transverse measure may be constructed from Lebesgue measure on a transverse circle to the foliated space. This passes to a trace on the foliation algebra which corresponds to the trace constructed above.

A projection $p$ is an element of $A$ that satisfies $p^{2}=p=$ $p^{*}$. Suppose that $p \in A$ is a projection. Then $0 \leq \tau(p) \leq 1$

\footnotetext{
${ }^{4}$ If you are an analyst you can think of $\mathcal{K}$ as the smallest $C^{*}$-algebra inside the space of bounded operators $\mathcal{B}(\mathcal{H})$ on a separable Hilbert space $\mathcal{H}$ that contains all of the operators with finite-dimensional range. If you are an algebraist at heart then you will be pleased to hear that $\mathcal{K}$ is the unique maximal ideal of $\mathcal{B}(\mathcal{H})$.
}

by elementary considerations. But what is the range of the map $\tau$ ? In the case $A=M_{n}(\mathbb{C})$ the range of $\tau$ would be the set $\left\{0, \frac{1}{n}, \frac{2}{n}, \ldots, 1\right\} \subseteq \mathbb{R}$. What happens for $A_{\lambda}$ ? Stay tuned.

To summarize, we have shown that the $C^{*}$-algebra $A_{\lambda}$ arises in three disparate arenas of mathematics. (There are others as well, but this should be enough to convince you that it happens a lot!) At this point, though, it is not at all clear to what extent the algebra is dependent upon $\lambda$. Let's find out.

\section{The World's Fastest Introduction to $K$-theory}

Suppose first that $A$ is a unital $C^{*}$-algebra. There are always projections, namely 0 and 1 . If $X$ is a connected space then these are the only projections in $C(X)$. On the other hand, $M_{n}(\mathbb{C})$ has lots of projections: for instance, take a diagonal matrix that has only ones and zeros on the diagonal. It turns out that $C(X) \otimes M_{n}(\mathbb{C})$ can have very interesting projections - these correspond to vector bundles over $X$.

Let $P_{n}(A)$ denote the set of projections in $A \otimes M_{n}(\mathbb{C})$, and define $P_{\infty}(A)$ to be the union of the $P_{n}(A)$ (where we put $P_{n}$ inside of $P_{n+1}$ by sticking it in the upper left corner and adding zeros to the right and below). Unitary equivalence and saying that $p$ is equivalent to $p \oplus 0$ puts a natural equivalence relation $\sim$ on $P_{\infty}(A)$. Then $P_{\infty}(A) / \sim$ has a natural direct sum operation, and we can turn it into an abelian group by doing the so-called Grothendieck construction (taking formal differences of projections). If you don't like that, take the free abelian group on the equivalence classes and then divide out by the subgroup generated by all elements of the form $[P \oplus Q]-[P]-[Q]$. This gives an abelian group denoted $K_{0}(A)$.

For example, take $A=\mathbb{C}$. Then $P_{n}(A)$ consists of all of the projections in $M_{n}(\mathbb{C})$. We learned in the second semester of linear algebra that every projection is unitarily equivalent to a diagonal matrix of the form $\operatorname{diag}(1, \ldots, 1,0, \ldots, 0)$. Hence, the equivalence classes of $P_{n}(\mathbb{C})$ are classified (via rank) by the integers $\{0,1,2, \ldots, n\}$, and the equivalence classes of $P_{\infty}(\mathbb{C})$ are classified by the natural numbers $\{0,1,2, \ldots\}$. Taking formal inverses, we obtain $K_{0}(\mathbb{C}) \cong \mathbb{Z}$. Note that the same answer emerges if we take $A=M_{j}(\mathbb{C})$ for any $j$, since "matrices of matrices are matrices."

For commutative unital $C^{*}$-algebras $A=C(X)$ with compact Hausdorff maximal ideal space $X$, the Serre-Swan theorem tells us that $K_{0}(C(X))$ is given by

$$
K_{0}(C(X)) \cong K^{0}(X)
$$

where $K^{0}(X)$ is the Grothendieck group generated by complex vector bundles over $X$.

We may regard $K_{0}$ as a functor on unital $C^{*}$-algebras and maps, since if $f: A \rightarrow A^{\prime}$ is unital, then $f$ takes projections to projections, unitaries to unitaries, and preserves direct sums. If $A$ is not unital, then we may form its unitization $A^{+}$(for example, $C_{o}(X)^{+} \cong C\left(X^{+}\right)$ where $X$ is locally compact but not compact and $X^{+}$is its one-point compactification), and then define $K_{0}(A)$ to be the kernel of the map

$$
K_{0}\left(A^{+}\right) \longrightarrow K_{0}\left(A^{+} / A\right) \cong \mathbb{Z} .
$$




\section{COMMUNICATION}

Since we are generally working with algebras tensored with the compact operators $\mathcal{K}$, it is good to know that $K_{0}(A) \cong K_{0}(A \otimes \mathcal{K})$.

Note that if $A$ is separable then there are at most countably many equivalence classes of projections, and hence $K_{0}(A)$ is a countable abelian group. Every countable abelian group may be realized as $K_{0}(A)$ for some separable $C^{*}$-algebra $A$.

\section{The $K$-theory of the Irrational Rotation}

\section{$C^{*}$-algebra: The Bad News}

Now, what is the $K$-theory of the irrational rotation $C^{*}$-algebra? A seemingly elementary question arises first: does $A_{\lambda}$ have any non-trivial projections? This was open for several years, and it led to decisive work by Marc Rieffel whose results, together with those of Pimsner and Voiculescu, we now describe. ${ }^{5}$

We are altering the historical order a bit in what follows.

If $\lambda$ is irrational then Pimsner and Voiculescu (1980) showed that

$$
K_{0}\left(A_{\lambda}\right) \cong \mathbb{Z} \oplus \mathbb{Z}
$$

independent of $\lambda$. This is just like for $A=C\left(T^{2}\right)$ ! So using $K_{0}$ by itself we cannot distinguish the various $A_{\lambda}$.

\section{Traces to the Rescue}

The key to distinguishing the family of $C^{*}$-algebras $\left\{A_{\lambda}\right\}$ is the trace defined at the end of the Homeomorphisms of the Circle section. If $A$ is a $C^{*}$-algebra with a normalized trace $\tau$ and $p$ and $q$ are orthogonal projections in $A$ (i.e. $p q=0$ ) then

$$
\tau(p \oplus q)=\tau(p)+\tau(q),
$$

and so the trace gives us a homomorphism

$$
\tau: K_{0}(A) \rightarrow \mathbb{R}
$$

of abelian groups. We have remarked previously that if $A$ is separable then $K_{0}(A)$ is a countable abelian group, and hence $\tau\left(K_{0}\left(A_{\lambda}\right)\right)$, the image of

$$
\tau: K_{0}\left(A_{\lambda}\right) \longrightarrow \mathbb{R},
$$

is a countable subgroup of $\mathbb{R}$. Pimsner and Voiculescu showed that the range of the trace

$$
K_{0}\left(A_{\lambda}\right) \cong \mathbb{Z} \oplus \mathbb{Z} \stackrel{\tau}{\longrightarrow} \mathbb{R}
$$

lies inside $\mathbb{Z}+\lambda \mathbb{Z}$, the subgroup of $\mathbb{R}$ generated by 1 and by $\lambda$. Rieffel showed that every element of $(\mathbb{Z}+\lambda \mathbb{Z}) \cap[0,1]$ is the image of a projection in $A_{\lambda}$. Combining these results gives us this omnibus isomorphism theorem:

Theorem 1 (Rieffel 1981 [2], Pimsner and Voiculescu 1980).

(1) If $\lambda$ is irrational then the image of the trace

$$
\tau: K_{0}\left(A_{\lambda}\right) \rightarrow \mathbb{R}
$$

is exactly $\mathbb{Z}+\lambda \mathbb{Z}$.

(2) If $\lambda$ and $\mu$ are irrational numbers in the interval $[0,1 / 2]$ with $A_{\lambda} \cong A_{\mu}$ then $\lambda=\mu$.

\footnotetext{
${ }^{5}$ It is easy to show that $A_{\lambda} \otimes \mathcal{K}$ has projections, and those projections determine the $K$-theory. It is a much deeper problem to deal with $A_{\lambda}$ itself.
}

(3) More generally, if $\lambda$ and $\mu$ are irrational numbers in the interval $[0,1 / 2]$ and $r$ and $s$ are positive integers with

$$
A_{\lambda} \otimes M_{r}(\mathbb{C}) \cong A_{\mu} \otimes M_{S}(\mathbb{C})
$$

then $\lambda=\mu$ and $r=s$.

(4) Suppose that both $\lambda$ and $\mu$ are irrational. Then $A_{\lambda} \otimes$ $\mathcal{K} \cong A_{\mu} \otimes \mathcal{K}$ if and only if $\lambda$ and $\mu$ are in the same orbit of the action of $G L(2, \mathbb{Z})$ on irrational numbers by linear fractional transformations.

So we see that the $A_{\lambda}$ retain all of the sensitive information about the angle $\lambda$. If we think back to the origins of $A_{\lambda}$ this seems really astonishing: The angle of the Kronecker flow deeply affects the geometry of the foliated space.

\section{References}

[1] C. C. MoOre and C. SchocheT, Global Analysis on Foliated Spaces, Second edition, Mathematical Sciences Research Institute Publications, 9, Cambridge University Press, New York, 2006. MR2202625

[2] M. A. RIEFFEL, $C^{*}$-algebras associated with irrational rotations, Pacific J. Math. 93 (1981), 415-429. MR623572

\section{Photo Credits}

Figure 1 courtesy of Andrea Gambassi and Corinna Ulcigrai. Author photo courtesy of Rivka Schochet.

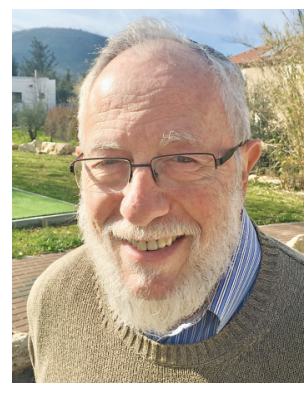

ABOUT THE AUTHOR

Claude (Chaim) L. Schochet mostly works at home in the tiny village of Bar Yochai, Israel looking out at Mt. Meron when he is not visiting local wineries or playing folk music on his guitar. We welcome visitors! 